Archives

$23 \mid 1999$

Officiers "moyens" (1)

\title{
Officiers « moyens » dans les enquêtes de 1573 et
} 1665

Jean Nagle

\section{Q OpenEdition}

\section{Journals}

Édition électronique

URL : http://journals.openedition.org/ccrh/2142

DOI : $10.4000 /$ ccrh. 2142

ISSN : $1760-7906$

Éditeur

Centre de recherches historiques - EHESS

Édition imprimée

Date de publication : 20 octobre 1999

ISSN : 0990-9141

Référence électronique

Jean Nagle, "Officiers «moyens » dans les enquêtes de 1573 et 1665 », Les Cahiers du Centre de Recherches Historiques [En ligne], 23 | 1999, mis en ligne le 17 janvier 2009, consulté le 30 avril 2019. URL : http://journals.openedition.org/ccrh/2142; DOI : 10.4000/ccrh.2142

Ce document a été généré automatiquement le 30 avril 2019

Article L.111-1 du Code de la propriété intellectuelle. 


\title{
Officiers « moyens » dans les enquêtes de 1573 et 1665
}

\author{
Jean Nagle
}

Il est plus qu'opportun, il est nécessaire de procéder à une définition très précise de l'office moyen lorsqu'on aborde des enquêtes où tous les offices sont confondus. Le dépouillement en devient plus souple et, semble-t-il, plus intéressant, dans la mesure où la notion d'officier moyen paraît fondamentalement adaptée à cette période de l'histoire de l'office'.

Examinons donc sérieusement comment l'adjectif «moyen » s'est finalement accolé à l'office, pour en revenir, si possible, à l'idée que les contemporains pouvaient se faire d'un tel groupe.

3 Partons d'un chapitre de Seyssel: "Du moyen Estat qui est le peuple gras " ${ }^{2}$ où la question est abordée sous l'angle des ordres, des états, et où l'on part d'une constatation de la dichotomie du tiers état :

A icellui appartient la marchandise [...] dont il peut faire grand acquêt pour autant qu'elle est du tout défendue au premier Etat de Noblesse [...]. A cettui Etat aussi appartiennent les offices des Finances, qui sont grands et de grand honneur et profit [...], les offices aussi de justice et de pratique de France, encores que les deux autres Etats en soient capables, toutefois ils sont communément et la plupart ès mains de cettuy Etat moyen.

Pour Seyssel, cet état moyen est un passage : ceux qui y sont tâchent toujours de parvenir au premier, qui est «le plus grand et le plus puissant outre qu'il est le plus digne». On peut certes parvenir naturellement " par vertu et par diligence ", du tiers état au second, mais il faut au second, pour atteindre à l'état de noblesse, le secours du roi ; on l'obtient assez facilement en cas de "grand service à la chose publique » : l'espoir d'ascension doit rester suffisant pour garantir la paix sociale.

4 Soixante ans plus tard, dans sa République (1579), fasciné par la cascade de la souveraineté, par la puissance de commander, Jean Bodin utilise la notion de «moyen » pour son analyse « essentielle» des avatars de la puissance, qui ne concerne, parmi les officiers, que les magistrats : 
Aussi pouvons-nous diviser les Magistrats en trois sortes pour le regard de la puissance : les premiers se peuvent appeler Magistrats souverains, qui ne doyvent obéissance qu'à la puissance souveraine ; les autres Magistrats moyens, qui doyvent obéissance aux Magistrats supérieurs et ont commandement sur autres Magistrats : les derniers sont ceux-là qui doyvent obéissance aux Magistrats supérieurs et ont commandement sur les particuliers ${ }^{3}$.

Bodin s'oriente ensuite vers l'étude des conflits d'autorité de ces magistrats entre eux.

Cette perspective, plus politique que sociale, n'engendre aucune nomenclature détaillée de ces magistrats, qui nous intéresserait bien ici ; c'est alors qu'on se tourne vers les œuvres de Charles Loyseau (1564-1627), qui, encore qu'ébloui par la société romaine, a traité exactement du point qui nous occupe pour la fin $d u X V I^{e}$ siècle : il faut seulement prendre soin de procéder à une lecture croisée du Traité des offices et du Traité des ordres. Lieutenant particulier au bailliage de Sens, puis bailli de Châteaudun pour Catherine de Longueville, Loyseau, qui s'est intéressé lui aussi au pouvoir de commandement des magistrats et des juges, a souligné en outre les gradations de l'honneur qui leur est dû, en donnant le détail de leurs titres.

Le terme magistrat ne signifie - chez les Latins - que leur plus noble office, scavoir est de ceux qui ont l'auctorité de commander, et ne comprend pas, ny les officiers ministres, ny les moyens comme ceux de finance qui ne sont ny Magistrats, ny ministres ${ }^{4}$.

Les «ministres» sont les auxiliaires de justice: «notaires, sergents, trompettes et autres », parmi lesquels les procureurs; les moyens sont justement notre gibier.

Les magistrats, qui ont le commandement, étant de ce fait sous la sauvegarde spéciale du prince souverain sont honorés et considérés comme sacrés et inviolables en tout temps et en tous lieux ${ }^{5}$. Ces magistrats sont proprement les seuls officiers dont la puissance est un " esclat et influence de la puissance absolue du Prince $»^{6}$, comme celle du prince n'est «qu'un rayon et esclat de la toute puissance de Dieu

[À ce titre, ces magistrats, et] principalement ceux du peuple fidelle, ont parfois des inspirations secrètes au fait de leur charge provenant de la grâce divine ${ }^{8}$.

Les simples officiers, non magistrats, quoique toujours en honneur, ne sont, eux, inviolables que dans l'exercice de leur charge.

7 Sont magistrats, pour Loyseau, ceux seulement qui ont pouvoir de justice et de commandement dans un territoire bien déterminé ${ }^{\prime}$ : donc les officiers des cours souveraines ordinaires, ceux des cours souveraines extraordinaires et les juges ordinaires, c'est-à-dire les baillis et sénéchaux au titre d'anciens juges, leurs lieutenants civils et criminels, généraux et particuliers ; les prévôts et leurs lieutenants ${ }^{10}$;

[...] quant aux avocats et procureurs du Roi des bailliages et des sénéchaussées, il y a

beaucoup d'apparence de les tenir pour magistrats parce qu'ils sont comme contrôleurs de justice ${ }^{11}$.

Ne sont pas magistrats : les conseillers présidiaux qui ne jugent

[...] qu'en troupe et compagnie ${ }^{12}$, non plus que

[...] les Esleus de France, officiers des gabelles, des Eaux et forêts, prévôts des

Maréchaux, juges consuls et tous autres juges extraordinaires ${ }^{13}$

Parmi ces « officiers médiocres », il faut compter encore

[...] entre autres presque tous les officiers de finance, esquels l'honneur prévaut ${ }^{14}$.

À la limite se situent « un tas de chétifs financiers » dont l'office « a peu d'honneur » ${ }^{15}$. Ils se différencient pourtant des « officiers ministres » dont

[...] les exercices dérogent à la noblesse, comme ceux de procureur postulant, greffier, notaire, sergents, clercs [qui sont] principalement fondés sur le gain ${ }^{16}$. 

d'avril 1997 (p. I et VII), et Maurice Gresset (p. 8), sont en gros, mais non absolument, d'accord avec Loyseau, puisqu'ils incluent dans les officiers moyens l'ensemble du personnel présidial.

9 À côté de sa position médiane entre l'office de magistrat et celui de ministre, Loyseau définit deux autres caractères du groupe moyen. Le premier, souligné déjà par Seyssel, est que

[...] ceux du second (état) tâchent toujours de parvenir au premier ${ }^{17}$.

La noblesse de ville, écrit Loyseau, a toujours tâché de se mêler et confondre avec les gentilshommes faisant profession des armes ${ }^{18}$ :

Les plus honnestes habitans des villes ayans dès longtemps pris coutume de se qualifier Nobles-hommes : celà a fait que ceux d'espée ont dédaigné ce titre, et se sont voulu qualifier Escuyer ${ }^{19}$.

Parmi ces « plus honnestes habitans »,

[...] tous officiers du Roy, et mesme les officiers de justice, ores que nos Royaux, bref tous officiers, hormis les ministres de justice ont prescrit ce titre et épithète de se qualifier Nobles-Hommes, pourveu toutefois qu'ils vivent noblement sans faire mestier ni marchandise,

puisque celle-ci est interdite en France aux gentilshommes par les états généraux. La qualité de noble-homme, à cause du degré de science qu'ils doivent avoir, convient mieux aux officiers de justice qu'aux autres ${ }^{20}$. De fait, au milieu du Xvi ${ }^{\mathrm{e}}$ siècle, écrit Bernard Chevalier, les magistrats des tribunaux locaux et quelques avocats se disent nobleshommes, les procureurs restant «honorables hommes ${ }^{21}$. Loyseau concédera que cette noblesse dont se qualifient les officiers de justice, communément appelée «noblesse de ville », n'est «qu'honoraire et de nom seulement » et n'apporte pas "les franchises et privilèges de Noblesse, comme l'exemption des tailles ${ }^{22}$. À la vérité, cette noblesse « est plutôt bourgeoise $»^{23}$. Pourtant elle est de la même nature que celle des «annoblis par dignités ", c'est-à-dire des officiers qui ont des offices anoblissants et peuvent atteindre personnellement à la « haute-noblesse $»^{24}$.

Une autre originalité soulignée par les textes de Loyseau, c'est la solidarité de l'office - de l'office moyen notamment - avec la ville. Celle-ci apparaît aux tenants de la noblesse de race comme un dangereux repaire d'officiers, témoin la diatribe d'Eutrapel, interprète de cette noblesse, à l'intention de l'avocat Lupolde :

Les villes certainement ont quelques beautés en nostre France, mais de nulle commodité que pour les gens de justice, marchands et artisans. Et se peut hardiment dire et asseurer quand l'on void un gentilhomme aux villes, qu'il y est appelant ou intimé, demandeur ou défendeur.

Il se débauche puis donne

[...] un coup de baston ou espée entre ses murs à quelque petit glorieux et rustre de ville qui l'avoit voulu braver : de là traîné et mangé en prison.

Vous Lupolde qui dès vostre jeunesse avez esté nourri aux villes, y plaidant et vivant des querelles d'autruy, n'estimerez jamais une retraite et vie champestre ;

et $\mathrm{Du}$ Fail critique le fait que les grandes charges, fonctions et gouvernements soient « entre les mains de nouveaux venus et tirez du populaire » ${ }^{25}$. Loyseau, à l'encontre, ne peut souffrir la noblesse rurale :

L'insolence des menus gentilshommes des champs est si grande [...] qu'il n'y a moyen de vivre en repos avec eux [...] ; ce sont des animaux sauvages, des oyseaux de proye [...] qui vivent de la substance d'autruy ${ }^{26}$. 
Ce thème a été largement développé dans la robe. Bertaut de Fréauville, qui sera conseiller au parlement de Paris, écrit, dans Les Prérogatives de la Robe (1701) :

Les gens du peuple devenus juges cessent d'être du corps du peuple. Il ne faut donc pas que les Nobles des campagnes qui sont issus de trois ou quatre races de gentilshommes et de chevaliers à lièvre [...] prétendent être au-dessus des Nobles des villes, qui sont issus de trois ou quatre races de conseillers et de présidents, à cause qu'ils ont trouvé moyen de s'exempter de la taille depuis cent cinquante ans en mettant une épée à leur côtée ${ }^{27}$.

11 Le lien des officiers avec la ville, dénoncé par la vieille noblesse, revendiqué par eux, est bien mis en valeur dans une classification des villes établie par les greffiers du Conseil du Roi, avec les tarifs du droit de marc d'or, à partir de 1603 au moins. C'est l'importance de son présidial qui détermine le statut de la ville : bonne, avec un bon présidial, ou un parlement, moyenne ou petite avec un moyen ou petit présidial ${ }^{28}$.

Les textes rassemblés ici sont bien connus, mais il faut souligner que l'abord des enquêtes de 1573 et 1665 a été heureusement transformé, quoique au prix d'un dépouillement plus délicat, par la prise en considération du groupe moyen, dont George Huppert avait pourtant plus qu'ébauché, dès 1977, l'originalité et le rôle, sans l'isoler toutefois suffisamment de la haute robe, ni insister assez sur la composante de l'office. C'est un renouvellement d'intérêt pour

[...] cette population limitrophe qui campe sur les confins de la noblesse, formant entre deux ordres une sorte de marche intermédiaire et qu'étudiait Jean-Richard Bloch,

[...] monde [continuait-il] qui sortait du peuple et n'en était plus ${ }^{29}$.

Examinons les éléments du groupe moyen dans les enquêtes de 1573 et 1665 . Celles-ci ont déjà été présentées ailleurs ${ }^{30}$; il n'est pas utile d'y revenir : 19400 offices sont recensés en 1573 (enquête incomplète), dont 8000 avec gages; 42300 en 1665. Pour ce premier dépouillement, je me suis intéressé uniquement aux pays d'élections: ressorts des parlements de Paris, partie de Dijon, partie de Bordeaux.

Les tarifs du marc d'or apportent une aide sérieuse au dépouillement; ces tarifs qui se fondent sur le « titre et qualité » de l'office, ou, plus tard, sur sa « qualité et mérite », ont été soigneusement complétés de 1583 à 1748, de sorte qu'on peut « catégoriser » presque tous les offices.

Un premier problème est de voir dans quelle mesure les greffiers du Conseil ont pris en compte, dans la classification, les données économiques ; c'est une lettre d'Henri III à son Conseil des Finances qui pose la question, le 28 septembre 1586, quand il demande à connaître la taxe des offices : « Pourquoi ne met-on pas à un prix tous les offices de même titre ? $»^{31}$. Les gages, dont nous disposons seuls pour 1573 , ne fourniront là-dessus que des réponses partielles.

Prenons l'exemple des présidiaux, pour les offices desquels la taxe du marc d'or est étroitement hiérarchisée selon le niveau du tribunal, lequel communique, rappelons-le, sa qualité à la ville; or, les gages ne s'ordonnent pas régulièrement de même. Pour le marc d'or, le lieutenant-général du bon présidial paie 324 l.t. ; celui du moyen : 216 l.t. ; celui du petit : 162 l.t., et le chef d'un siège ressortissant nuement au parlement : 108 l.t. $\mathrm{Au}$ bon présidial, le lieutenant-criminel, le procureur du roi et le greffier cotisent 216 l.t., comme le lieutenant-général d'un moyen siège, c'est-à-dire un marc d'or, qui, suivant notre hypothèse, est déjà un marc d'or de noblesse. Mais les gages, à la différence du marc d'or, ne varient guère, pour un office donné, d'une catégorie de présidial à l'autre. Si la 
moyenne des gages des offices d'un bon présidial est de $6 \%$ supérieure à celle de l'ensemble des présidiaux, celle du moyen présidial y est de $6 \%$ inférieure, celle du petit présidial est de $10 \%$ supérieure - surpassant donc celle du bon présidial, alors que le siège royal est à équivalence avec le moyen présidial. Les lieutenants-particuliers des sièges présidiaux et royaux, dont la taxe pour le marc d'or impose logiquement quatre cotes : 189, 135, 108 et 54 l.t., touchent, dans les quatre cas, les mêmes cent livres de gages. Cela montre que le principe du début d'un siècle où l'on taxait la confirmation "selon la qualité des offices et valeur des gages ", ainsi que le dit Barillon dans son Journal (t. I, p. 4), reste valable. Il est clair qu'il faut prendre aussi en compte, avec les droits et les épices, la finance de l'office. D'après notre exemple : qualités différentes, mêmes gages, on considérera que, pour les offices de justice proprement dits, à la fin du XVI ${ }^{\mathrm{e}}$ siècle, le titre l'emporte sur les gages. Il n'en va pas de même pour les petits juges extraordinaires, pour les élus de France, les grenetiers, les maîtres particuliers des eaux et forêts, dont la cotisation pour le marc d'or est proportionnelle aux gages, ce qui montre que l'office en soi est sans qualité, le montant étant toujours le résultat de l'application de la formule de Barillon. Ce ne sera plus le cas pour les receveurs, dont le marc d'or est fixé directement sur la taxe de leurs offices - sa valeur économique - sans plus tenir compte des gages qui sont artificiellement élevés :

Il n'y a si petit exercice de finance [dit Loyseau] dont on n'ait fait un office. Et pource qu'il y a ordinairement peu d'honneur et peu de pouvoir en ces offices, on leur attribue beaucoup de gages ${ }^{32}$.

Dans les tarifs du marc d'or, le niveau des officiers moyens se situe entre les cotes 40 l.t. 10 s. et 108 l.t. Dans la hiérarchie locale, on trouve d'abord, au degré le plus élevé, des charges qui ont un rapport avec le niveau supérieur de l'office : le bailli, le lieutenantgénéral (216 l.t. en moyenne); au second étage, le lieutenant-criminel, le procureur du roi, et le greffier civil et criminel - malgré Loyseau qui noie cette dernière fonction parmi celles des officiers ministres - enfin le lieutenant-particulier : cote de 108 à 189 l.t., selon la dignité de la ville. Au-dessous sont les offices proprement moyens : à 108 livres on trouve le président du présidial, l'avocat du roi, les conseillers, les receveurs généraux ; à 81 l.t. en moyenne, le prévôt, le juge, le receveur des tailles, des aides, du taillon de l'élection; le prévôt des maréchaux, le maître particulier des eaux et forêts ; vers 67 l.t., les contrôleurs généraux du taillon, les contrôleurs provinciaux, les élus et contrôleurs des tailles, les grenetiers et lieutenants des greniers à sel, les lieutenants des prévôts des maréchaux ; à 54 l.t., les greffiers des maréchaussées et des élections, les procureurs du roi des élections, à 40 l.t. enfin les avocats, procureurs du roi et greffiers des eaux et forêts. Ici finit le niveau moyen; ceux que l'on trouve en dessous sont les ministres de justice.

18 Pris globalement, les gages apportent quelque vérification à la qualification hiérarchisée des institutions : si nous considérons en gros les présidiaux de nos ressorts, par exemple la somme moyenne des gages plutôt que la moyenne individuelle, l'ensemble des gages, pour les 43 présidiaux concernés monte à 101567 l.t. pour un total de 825 officiers gagés. Pour les bons présidiaux, la somme des gages est de 3300 l.t., pour un personnel gagé de 27 ; les moyens présidiaux ont un total de 2300 l.t. pour 18 officiers; les petits, 1800 livres pour 15 personnes; les officiers les plus élevés représentent un peu plus du cinquième des effectifs et le quart des sommes; les officiers moyens gagés la presque totalité du reste. Il faut naturellement évoquer en outre la présence, autour de ces 
tribunaux, d'une nébuleuse de 2000 à 3000 auxiliaires de justice : sergents, notaires et procureurs.

19 Les tribunaux de justice extraordinaires se sont développés, à cette époque, après le remplissage des postes de tour: alternatifs, voire triennaux, par la création de charges presque purement honorifiques, très peu techniques : présidents, assesseurs, etc. Il est probable que l'affaiblissement final du groupe provient largement de cette surcharge parasitaire.

En ce qui concerne les 103 élections de 1573 et leurs 40 élections particulières, le personnel atteint 1012 officiers, dont les gages montent à 292163 l.t., ainsi répartis : 106718 l.t. pour les 381 élus, c'est-à-dire $37 \%$ de la somme comme des effectifs ; 21812 l.t. pour les 86 contrôleurs, soit 7,5\% de la somme pour $8,5 \%$ des effectifs ; et 141587 l.t. pour les 266 receveurs : $48 \%$ de la somme pour $27 \%$ des effectifs. Le nombre des élections s'accroît de moitié au XVII ${ }^{\mathrm{e}}$ siècle, jusqu'à 164 et 30 particulières, et les effectifs gagés vont doubler (2000); le nombre des élus augmente des deux tiers ; celui des receveurs de $20 \%$ seulement (332 en 1665 contre 266 en 1573); le principal accroissement, en dehors de l'extension du système de l'élection aux généralités de Montauban et de Grenoble, est dû à l'inflation du nombre des présidents (255), des lieutenants (178) et des assesseurs (144), soit un nouveau sous-groupe d'officiers équivalent à celui des élus (577 contre 594), dont on peut penser qu'il a profondément modifié l'esprit des élections.

21 Les greniers à sel, en 1573, sont au nombre de 148, avec un personnel de 900 membres dont 232 grenetiers et 216 contrôleurs; on note la différence avec les élections, où les contrôleurs étaient quatre fois moins nombreux que les élus. La somme totale des gages est de 60000 l.t. Les grenetiers en touchent $60 \%$, les contrôleurs $28 \%$; ceux-ci, presque aussi nombreux que ceux-là, ont des gages deux fois moins élevés. Le plus souvent le procureur du roi est commun à l'élection et au grenier à sel; le greffier du sel touche le plus souvent 20 livres de gages ; le greffier de l'élection, quand il est gagé, reçoit deux ou trois fois plus. Dans les gabelles aussi, à côté des charges de tour, l'hypertrophie s'est portée à la tête, par un développement de la présidence.

22 Pour les autres juridictions extraordinaires, les offices moyens se sont adaptés à des structures archaïques, souvent issues de la féodalité ou des grands apanages, mais, par une sorte de paradoxe, ils se sont développés surtout par une simple duplication, une colonisation méthodique de proche en proche.

En 1573, les effectifs des officiers des eaux et forêts sont de 250, le double avec les sergents. Il y a 52 maitres particuliers et 62 lieutenants; les maîtres touchent 300 à 400 livres de gages, les lieutenants quatre fois moins ; le fait le plus notable est ici la disparité régionale : moyenne en Champagne, Bourgogne, Berry, Touraine, la densité de ces offices devient très forte en Normandie : 110 officiers, non compris presque 200 sergents; les effectifs sont multipliés par trois en 1665 (1 400 officiers). On compte en France, en 1573, 32 prévôts des maréchaux, touchant chacun 600 livres de gages et payant 162 livres de marc d'or, soit comme le lieutenant-criminel d'une ville "moyenne", qui touche, lui, 120 l.t. de gages. Le lieutenant du prévôt paie 108 l.t. de marc d'or, pour la moitié moins de gages. Il y a alors 45 lieutenants en tout ; plus de la moitié des officiers sont stationnés dans l'ouest et le centre ouest: Normandie, Touraine, Poitou (45 sur 97); les archers, au nombre de 400, sont tous bien gagés : 180 livres chacun, pour 20 livres de marc d'or; le tiers de ces archers est basé en Touraine. L'effectif total de la maréchaussée est donc de 
600 à 700 officiers. En 1665, il sera de 3300 hommes, ce qui traduit pourtant un recul en pourcentage : de $8,4 \%$ à $7,6 \%$ du total des offices de ce type.

En définitive, on rencontre ces officiers moyens que nous venons d'examiner, en 1573, dans les :

\begin{tabular}{|c|c|c|}
\cline { 2 - 3 } \multicolumn{1}{c|}{} & Effectifs & Gages (l.t) \\
\hline Présidiaux & 640 & 75000 \\
\hline Elections & 733 & 270617 \\
\hline Greniers à sel & 248 & 54000 \\
\hline Eaux et forêts & 114 & 35000 \\
\hline Maréchaussée & 80 & 30000 \\
\hline Total & 1815 & 4164617 \\
\hline
\end{tabular}

Disons, en gros, 2000 officiers moyens, pour 500000 livres de gages ; ils équilibrent une masse de gages comparable qui concerne les officiers supérieurs des cours souveraines et bureaux des finances du ressort concerné : parlements de Paris, Bordeaux, Rouen, Dijon ; cours des aides de Paris, Rouen, Montferrand ; chambre des comtes de Paris.

Pour le détail :

\begin{tabular}{|c|c|c|}
\cline { 2 - 3 } \multicolumn{1}{c|}{} & Effectifs & Gages (1.t) \\
\hline Cours souveraines & 429 & 341070 \\
\hline Bureaux des finances & 81 & 131927 \\
\hline Total & 510 & 472997 \\
\hline
\end{tabular}

Il y a donc quatre fois moins d'officiers supérieurs que d'officiers moyens; selon l'enquête de 1573, il n'y aurait qu'une demi fois moins d'officiers moyens que de "ministres » gagés : il reste donc à estimer l'effectif de l'ensemble des auxiliaires, y compris les nongagés, en établissant des coefficients selon les différents types de juridictions; il est probable qu'ils surpassent deux ou trois fois le nombre des moyens; il y aurait aussi à établir, à propos de ces officiers moyens ou ministres, y compris les avocats, la proportion de ceux qui sont titulaires d'une fonction judiciaire seigneuriale, le taux de cumul et le nombre des officiers purement seigneuriaux ${ }^{33}$.

Le groupe des nobles-hommes n'a pas été éternel. Après avoir évoqué les épithètes d'honneur convenables pour les clercs (discrète personne), les officiers (noble-homme), les bourgeois (honorable homme), Loyseau indique :

C'est la science des Secrétaires de scavoir discerner les épithètes qu'ils peuvent attribuer à chacun ordre et qualité de personnes ${ }^{34}$.

Vers 1750 , d'Alembert considère, au contraire l'épithète de noble-homme comme tout à fait surannée :

C'est aux généalogistes à nous apprendre le sens précis de cette expression, surtout dans certaines provinces ${ }^{35}$.

Ce n'est pas sa simple position médiane qui a défini le groupe moyen, c'est un état d'esprit, une fonction, des modes techniques d'exercice, bref, des caractères propres et donc transitoires.

Le premier trait à souligner est peut-être l'opposition, en ce qui concerne la carrière, entre la fixité des officiers moyens et la mobilité des officiers souverains : Chasseneuz, Poyet, La Guesle, Verdun, Le Jay, ont voyagé d'un parlement à l'autre ; mis à part certains 
lieutenants-généraux surtout, l'exercice des officiers moyens s'est maintenu dans un cadre local.

Ils font partie cependant de la sphère d'intérêt du pouvoir, et sont, comme les officiers militaires, matière à notation ${ }^{36}$. Dans les enquêtes provinciales de 1665 , les intendants recueillent des renseignements sur ces officiers moyens, sur leur fortune (dettes, richesse), sur leur moralité (débauche, violence), sur leur influence et leur «manière de servir ${ }^{37}$; de tels renseignements ne seront réunis ni sur les auxiliaires de justice ni sur les bas-officiers des armées.

Le rôle du groupe moyen est sans doute d'une certaine manière comparable à celui que Halbwachs a dévolu à une partie de la classe moyenne,

[...] définie par son activité technique et la connaissance pratique des règlements à appliquer sûrement,

activité en partie "mécanique ", la fonction d'adaptation étant confiée à la classe supérieure. Les classes moyennes, pour Halbwachs, se définissent plutôt comme une catégorie de population urbaine, de petites villes, autrefois constituée, dit-il,

[...] par le personnel subalterne des administrations royales, seigneuriales, provinciales et municipales, et aujourd'hui par

[...] le haut-artisanat, les petits et moyens commerçants et industriels, une partie des professions libérales, les fonctionnaires moyens; la conscience paysanne resterait première dans ces classes moyennes ${ }^{38}$.

À la lecture du Conseiller d'État de Philippe de Béthune (1631), on se rend compte qu'il était, pour lui, admissible, voire souhaitable, que les officiers moyens aient des qualités moyennes: il vaut mieux choisir pour les affaires, dit-il, des gens «de médiocre entendement pourveu qu'ils soient gens de bien »; ils supportent plus facilement les accidents qui surviennent, étant ordinairement flegmatiques, que ceux qui ont « une aigüe subtilité ", et sont généralement " cholériques », désireux de nouveautés, et qu'il y a finalement intérêt à faire passer d'une charge à l'autre, en leur donnant "plus d'honneur et moins de pouvoir $»^{39}$.

Les juges extraordinaires devaient avoir quelque instruction, les juges ordinaires étaient gradués et passaient, avant d'être pourvus, un examen de droit ; l'instruction accessible localement est une des préoccupations de nos officiers, comme l'a très bien souligné George Huppert ${ }^{40}$. Les tenants de la noblesse de race incriminent le développement des collèges, en accusant la politique de François $\mathrm{I}^{\mathrm{er}}$ :

Il ne fut jamais une plus fausse politique que celle de ce roi [écrit le sieur de Caillière] ; il remplit les barreaux de chicaneurs et d'avocats, les villes de fainéans et les cloîtres de moines ${ }^{41}$.

Ou encore, pour le sieur Pelletier, en 1615, les études font l'homme de robe longue; devenu officier celui-ci répudie l'enseigne, la boutique, le chaperon de la grand'mère : «Adieu les petites gens! »; et les villes

[...] se remplissent d'une effrenée multitude d'estudians et de luxe et d'oisivetét ${ }^{42}$. Toutefois le programme culturel des officiers moyens n'a pas l'ambition de celui de la grande robe, et se marque déjà par une distance à l'égard de l'antiquité, que l'on retrouvera chez les ingénieurs militaires. Pour Nicolas Pasquier, il n'y a pas lieu de verser dans les études latines et grecques :

La langue maternelle peut [...] à suffisance communiquer toute doctrine, parce qu'il n'y a discipline propre au gentilhomme [...] qui ne soit doctement et dissertement traictée en notre langue ${ }^{43}$. 
Pierre Charron, dans La Sagesse, reconnaît bien " toute la grâce et énergie » des citations latines, mais son parti pris est tout différent de celui de Montaigne :

Je traicte et agis icy non pédantesquement; je parsème des sentences latines très courtes, fortes et poétiques tirées de très bonne part, et qui n'interrompent ny troublent le fil du texte français ${ }^{44}$.

Les mathématiques, conseille Pasquier, seront cultivées parce qu'elles rendent « l'esprit prompt, vif et aigu $»^{45}$; intérêt très légitime dans un groupe qui reste lié à la marchandise, et où se fait jour la volonté de donner la "suffisance ", c'est-à-dire une compétence reconnue, aux comptables, dont la fonction serait nettement séparée de celle des secrétaires; tout en exposant ce souhait, Antoine de Laval décrit les soucis du receveur, dont il s'agit de renforcer l'indépendance par le prestige technique :

C'est une dure et serve condition [dit-il] que d'être petit contable hors de la cour, car là, un homme trouve toujours quelque puissant parrain qui le met à l'abri de son autorité. Et puis chez le roi, il n'est point de petit office, mais en quelque autre lieu que ce soit, les contables ont de grandes alarmes, de grands soucis et d'étranges servitudes : partant n'en conseillerai-je jamais la condition [...] à ceux que j'aime ${ }^{46}$.

Ce programme moyen, sans référence explicite à l'exemple de l'héroïsme antique, vise à l'usage de la raison, à la recherche, dit Pasquier, d'une " philosophie des mœurs, qui aide à conduire sa vie au bien $»^{47}$. Caillière, pour le noble d'épée, se contentera d'une «science du monde ", d'une "prudence ", qui puisse guider son élève vers la faveur ${ }^{48}$. Charron, dont le livre a eu tant de succès dans la robe, parle, lui, d'une vertu associée à « la science, la justice, la bonté, la beauté, les richesses ", et qui permette d'accéder à la "noblesse acquise et personnelle » qui « provient de l'esprit et non du sang ${ }^{49}$.

C'est Charron encore qui trouvera des mots définitifs pour répondre à Du Fail, ou du moins à Eutrapel, à propos de l'incapacité des gens de robe à apprécier la campagne : il souhaite se dégager des préoccupations de la ville, de ses affaires, de ses querelles :

La vie rustique est bien plus nette, innocente et simple [...]. Vivre aux villes, c'est estre au monde banni et forclos du monde, et il évoque les anciens officiers qu'on

[...] tirait de la vie rustique, et y retournent ayant achevé leur charge ${ }^{50}$.

Les officiers moyens ont du reste largement participé au mouvement littéraire de retour à la terre qu'on a heureusement baptisé "Révolution bucolique $»^{51}$ : que ce soit Germain Forget, avocat au présidial d'Evreux; Claude Binet "Magistrat ", Les Plaisirs de la vie rustique et solitaire, 1583 ; Robert Angot, conseiller au présidial de Caen; Isaac Habert, commissaire de l'artillerie ; Philibert Guide ou Nicolas Rapin, vice-sénéchal du Bas-Poitou à Fontenay-le-Comte, Les Plaisirs du gentilhomme champestre, 1581. Sans prétendre que ces officiers aient eu une contribution très originale à cette "révolution ", on peut noter pourtant que les descriptions de Forget ou de Binet offrent des traits réalistes, concrets, qui ne laissent pas place à l'idéalisation et vont à l'essentiel ; Claude Binet décrit par exemple le repas d'un couple de paysans, Janot et Fleurie ; pour leur déjeuner et celui de leurs enfants, ils préparent des rôties grasses : la graisse du lard qui rôtit s'égoutte sur les tranches de pain grillé, l'odeur se répand,

Mais Janot se reprend: Il faut, dit-il, premier Dieu l'autheur de tout bien dévotement prier ${ }^{52}$.

À la fin d'une journée de moisson, évoquée par Claude Binet, on voit les paysans « recrus, suants d'ardeur ", et " au non-pâle teint » regagner leurs « obscures tanières »; ils vont adoucir « l'aigreur de toute la journée » avec leur « pain demi-brun », leurs «peu friands bruvages », un brouet «commensal » (est-ce à dire partagé, et peut-être chiche ?), le 
«fromage doüillet », et «l'Attique festin afranchy de fumée » (du miel sans doute), avant de regagner « de [leurs] licts enfumez le trop ferme duvet $»^{53}$. Ici la matière poétique est la chose vue, sans transposition, sans interposition de l'antique. Le regard de l'officier moyen, limité, mais aussi éduqué par le jeu dialectique du pour et du contre, aurait donc eu, semble-t-il, ses propres lumières.

Pour conclure logiquement sur les enquêtes, il reste à dépouiller, notamment en ce qui concerne les présidiaux, l'enquête de 1665 , et les deux textes pour les pays d'État; ces résultats se trouveront dans un ouvrage en collaboration sur les offices à paraître sous peu.

\section{NOTES}

1. Michel Cassan, éd., Les Officiers « moyens » à l'époque moderne, Limoges, Pulim, 1998, 399 p.

2. Claude de Seyssel, La Monarchie de France et deux autres fragments politiques, (1519), Jacques Poujol, éd., Paris, Librairie d'Argences, 1961, 253 p., p. 128. La notion de « moyennes personnes » entre «petits» et «nobles puissants et riches» existe chez Pierre de Crescens, Livre des proffits champestres, Paris, Chavane, 1965, p. 47. Philippe de Béthune, dans Le Conseiller d'État (1632) : « en toute sorte d'État il y a trois sortes de personnes, les grands qui ont puissance, crédit, richesses ; les pauvres et misérables qui ont nécessité de tout, et les médiocres; les derniers sont ordinairement plus paisibles à gouverner », chap. XIV, p. 436.

3. Jean Bodin, La République (1579), 6 vol., Paris, Fayard, 1986, liv. III, chap. III, p. 90 ; chap. VI, p. 145.

4. Charles Loiseau, «Traité des offices ", Euvres, Genève, Philippe Albert, 1620, liv. I, chap. I, § 7, p. 7.

5. Ibid., chap. VI, § 14, p. 90.

6. Ibid., chap. v, § 8, p. 88.

7. Ibid., liv. II, chap. II, p. 260.

8. Ibid., liv. I, chap. v, § 5, p. 88.

9. Ibid., liv. I, chap. v, $\S 48$, p. 96

10. Ibid., chap. VI, §54-55, p. 97.

11. Ibid., § 63, p. 98 .

12. Ibid., §56, p. 97.

13. Ibid., $\$ 47$, p. 96 .

14. Ibid., § 3, p. 88.

15. Charles Loiseau, « Traité des ordres », CEuvres, op cit., chap. XI, § 27, p. 177.

16. Charles Loiseau, «Traité des offices », op. cit., liv. I, chap. VI, § 3, p. 88 ; chap. V, § 106, p. 83.

17. Claude de Seyssel, op. cit., liv. I, chap. XV, p. 123.

18. Charles Loiseau, «Traité des ordres », op. cit., chap. v, § 20, p. 66.

19. Ibid.

20. Id., « Traité des offices », op. cit., liv. I, chap. vIII, § 61, p. 120.

21. Bernard Chevalier, Les Bonnes Villes, Paris, Aubier, 1982, 344 p., p. 147.

22. Charles Loiseau, «Traité des ordres », op. cit., chap. XI, § 13, p. 174.

23. Id., «Traité des offices », op. cit., liv. I, chap. vIII, § 62, p. 120. 
24. Id., « Traité des ordres ", op. cit., chap. IV, § 44-45, p. 61.

25. Noël du Fail, Contes et discours d'Eutrapel (1575), 2 vol., Paris, Éd. Chippeau, 1875, t. II, p. 272 sq.

26. Charles Loiseau, «Traité des ordres », op. cit., chap. v, § 69, p. 76.

27. Livre cité dans le texte, p. 283.

28. Jean Nagle, Le Droit de marc d'or des offices. Tarifs de 1583, 1704, 1718. Reconnaissance, fidélité, noblesse, Genève, Droz, "Travaux d'histoire éthico_politique, n ${ }^{\circ} 52$ », 1992, 277 p., p. 252. Les cotes du marc d'or citées ci-dessous proviennent de ces tarifs.

29. George Huppert, Bourgeois et gentilshommes, Paris, 1983, 216 p.; Jean-Richard Bloch, L'Anoblissement au temps de François Premier, Paris, 1934, 216 p.

30. Enquête de 1665 : Jean Nagle, Histoire de la fonction publique en France, Marcel Pinet (dir.), t. II, Paris, Nouvelle librairie de France, 1995, 3 vol., p. 185-187. Enquête de 1573 : id., Bulletin de la société d'histoire moderne, $\mathrm{n}^{\circ}$ 3-4, 1994, p. 27-34.

31. Noël Valois, Inventaire des arrêts du Conseil d'État, 1886, t. I, p. LVIII, publication de BnF, Ms. Fr. 16233, fos $470-473$.

32. Charles Loiseau, «Traité des ordres ", op. cit., chap. VIII, § 84, p. 134.

33. Frédéric d'Agay évalue à 70000 les juges seigneuriaux : Philippe Boucher (dir.), La Révolution de la justice, Paris, Jean-Pierre de Monza, 1989, 273 p., p. 35.

34. Charles Loiseau, «Traité des ordres », op cit., chap. XI, § 37, p. 180.

35. D'Alembert, Éloges académiques : Fléchier. Cité par Littré, Dict., t. V, « noble », nº 2.

36. BnF, Ms. Fr. 4255 : Observations sur les officiers de cavalerie et d'infanterie.

37. BnF, Ms. Cinq cents de Colbert, 279. Enquête de l'intendant de Moulins, Pomereu, 1665.

38. Maurice Halbwachs, "Les caractéristiques des classes moyennes ", 1939, repris dans Classes sociales et morphologie, Paris, Éditions de Minuit, 1972, 463 p., p. 95-111.

39. Philippe de Béthune, Le Conseiller d'État, 1632, p. 140.

40. George Huppert, op. cit., p. 102-155 ; Roger Doucet, Les Bibliothèques parisiennes au XVI siècle, Paris, 1956, $175 \mathrm{p}$.

41. Caillière, Le Maréchal de bataille, Paris, 1658, p. 371.

42. Pelletier, L'Image de la France, Paris, 1615.

43. Nicolas Pasquier, Le Gentilhomme, Paris, 1611.

44. Pierre Charron, La Sagesse (1601), Paris, Bastien, 1783, p. XxVII.

45. Nicolas Pasquier, op. cit.

46. Antoine de Laval, Desseins des professions nobles et publiques, Paris, L'Angelier, 1605, fol. $308 \mathrm{v}^{\circ}$.

47. Nicolas Pasquier, op. cit.

48. Caillière, op cit., p. 375.

49. Pierre Charron, op. cit., liv. I, chap. LIV, § 5, p. 280.

50. Ibid, chap. LII, p. 270.

51. Françoise Joukovsky, La Renaissance bucolique, 1550-1600, Paris, Flammarion, 1994, 271 p.

52. Ibid., p. 105.

53. Ibid., p. 64. 